\title{
Infrastructure-Assisted Geo-Routing for Cooperative Vehicular Networks
}

\author{
D. Borsetti and J. Gozalvez \\ Ubiquitous Wireless Communications Research Laboratory \\ Uwicore, http://www.uwicore.umh.es \\ University Miguel Hernández, Elche, Spain \\ dborsetti@umh.es,j.gozalvez@umh.es
}

\begin{abstract}
Cooperative vehicular systems require the design of reliable and efficient multi-hop networking protocols to achieve their foreseen benefits. Although many geo-routing protocols have been proposed in the literature, few contributions have analysed the benefits that road side infrastructure units could provide to successfully route data from source to destination. In this context, this paper proposes a novel infrastructure-assisted routing approach designed to improve the end-to-end performance, range and operation of multi-hop vehicular communications by exploiting the reliable interconnection of infrastructure units. The conducted investigation shows that the proposed infrastructureassisted routing approach achieves its objectives, and reduces the routing overhead compared to other greedy position-based geo-routing protocols. Finally, the paper shows that to obtain the maximum benefits from the proposed infrastructure-assisted routing approach, optimal infrastructure deployment strategies must be further investigated.
\end{abstract}

Keywords: cooperative vehicular systems, vehicular adhoc networks, road side infrastructure, routing protocols, multi-hop communications.

\section{INTRODUCTION}

Cooperative vehicular communications have attracted the interest of the research community due to its potential benefits (traffic safety, traffic management, and infotainment), and important technical challenges. The provision of the foreseen V2X (Vehicle-to-Any communications) services will largely depend on network connectivity, and hence, on vehicular density and infrastructure deployment. As a result, two major challenges for the successful deployment of cooperative vehicular communications are the design of reliable multi-hop communications and networking techniques, and the design of efficient solutions to overcome the gradual introduction of cooperative V2X technologies in vehicles. Although a large number of multi-hop routing and data dissemination studies have been published in the literature, the challenging propagation conditions, in particular for Vehicle-to-Vehicle (V2V) communications, and the highly dynamic vehicular networks still represent important difficulties to provide reliable multi-hop communications over distant vehicles. In addition, a smaller number of studies have addressed the gradual introduction of V2X technologies, and proposed technological solutions that can provide the levels of network connectivity required by many V2X services. In this context, the deployment and use of road side infrastructure units can provide interesting solutions to address some of the cooperative vehicular networking challenges.

Several studies have recently explored the use of Road Side infrastructure Units (RSUs) in cooperative vehicular systems. In [16], Lochert at al. investigate the performance of a stub dissemination protocol during the roll-out phase of Vehicular Ad-hoc NETworks (VANETs). In order to deal with a low market penetration rate, the authors propose to install RSUs to support the dissemination process. The authors also consider in [17] a cooperative traffic information system where vehicles gather information on traffic condition, and diffuse them in order to improve route planning. Besides proposing a data aggregation mechanism to limit the required overall bandwidth, the authors consider the use of infrastructure units for the early deployment stage, and present a genetic algorithm to optimally locate the RSUs in order to speed up the dissemination process. In [23], Zhao et al. address the data dissemination problem in VANETs, and propose a data pouring scheme that tries to optimize the network dissemination capacity by selecting a subset of main roads, called axis roads, where the information has to be poured. The authors propose to install road side units at the intersections of axis roads acting as relay and broadcast stations. Similarly, in [7], the authors introduce the concept of wireless dead drops to accumulate and exchange data with passing vehicles. The work presented in [20] also deals with information dissemination in VANETs. The authors consider an urban scenario where infrastructure units are employed to disseminate information to vehicles, and formulate an optimization problem to find the optimal deployment of the infrastructure units in order to maximize the number of informed vehicles. In [1], Aslam et al. start from the consideration that $\mathrm{V} 2 \mathrm{~V}$ communications are not always feasible when a low market penetration rate is considered. The authors suggest then to complement the lack of V2V communications by using partially connected RSUs, and propose a store and forward mechanism where messages are opportunistically carried by vehicles travelling between RSUs. The contributions reported in [5], [3], and [19] also consider Vehicle-to-Infrastructure (V2I) communications, but are focused on the problem of providing Internet connection to vehicles. In this context, RSUs are used as a gateway to Internet, and not as a complement to the vehicular adhoc network. In [11], the authors exploit RSUs to provide multiple link-disjoint paths between source and destination, 
and therefore improve the reliability of $\mathrm{V} 2 \mathrm{~V}$ communications, considering a highway scenario where RSUs are uniformly deployed. To this aim, the authors consider the concept of closed sector, introduced in RAR [18], to route packets among vehicles; RAR is further discussed in the next paragraph.

The discussed contributions succeed in exploiting RSUs to provide more ubiquitous connectivity in vehicular networks. However, they do not tend to consider the potential benefits that RSUs can offer to improve the reliability of end-to-end multi-hop vehicular communications by assisting geo-routing protocols. RSUs present two major potential advantages for multi-hop vehicular communications. In the first case, the higher antenna height increases the range and reliability of V2I communications in comparison to V2V communications. In addition, RSUs will be connected to reliable backbone networks to enable traffic authorities the centralised access, configuration and maintenance of these units. As a result, RSUs can be considered to be directly connected to each other, independently of their geographical distance, which provides a valuable opportunity to improve the reliability and range of multi-hop vehicular communications. In this context, it is important to highlight two major contributions investigating the impact of RSUs on the routing process in vehicular networks. The first contribution, referred to as Roadside-Aided routing protocol (RAR), was presented in [18]. In RAR, the geographical area of interest is partitioned into closed sectors formed by RSUs placed at the extremities of such sector.

The authors propose then a protocol that manages to efficiently route packets among vehicles of different sectors through the use of RSUs. The authors show that RAR is able to provide good routing performance by geographically scaling the considered scenario. Although interesting, the RAR protocol requires the deployment of a large number of RSUs to form the closed sectors, and ensure a high packet delivery ratio, which could compromise its feasibility, in particular during the roll-out phase of cooperative vehicular systems. Moreover, even if the RAR protocol doesn't require hierarchical addressing, it makes use of an affiliation protocol in order to associate vehicles to closed sectors. Conversely, the approach proposed in this paper aims to harmonize the infrastructure and the vehicular ad hoc network in a transparent fashion without adding undesirable overhead.

In [2], the authors investigate a geographic routing approach that considers either pure V2V multi-hop communications, or hybrid $\mathrm{V} 2 \mathrm{~V}$ and $\mathrm{V} 2 \mathrm{I}$ communications. A metric is then introduced to select the optimal communications path between source and destination. However, to achieve its objective, the proposal relies on updated information about the topology of the ad-hoc network. In their proposal, the authors consider that before sending a packet, the source node is aware of the number of hops needed to reach the destination through either the pure V2V or hybrid V2V-V2I communication links. To obtain this information, a discovery service is necessary, which would then increase the complexity and routing overhead given the dynamic nature of vehicular communications. In this context, this paper proposes a novel infrastructure-assisted routing approach designed to improve the reliability and operation of multi-hop vehicular communications by exploiting the reliable interconnection of RSUs. The proposed approach is not based on a novel route selection metric, but a novel graph representation of the road topology. In addition, it does not require an initial large deployment of RSUs to start improving the reliability of vehicular communications, or a route service discovery process, which considerably reduces its complexity and ensures its successful operation even under very dynamic vehicular scenarios.

The rest of the paper is organized as follows. Section II introduces geo-routing in vehicular networks, and presents the infrastructure-assisted routing approach proposed in this paper. Section III presents the simulation scenario, and Section IV analyzes the performance of the proposed approach. Finally Section V concludes the paper.

\section{GEO-Routing FOR VEHICUlar Networks}

A significant number of routing protocols for vehicular adhoc networks have been proposed and evaluated by the scientific community in recent years. Geographic greedy forwarding approaches have been proven to be particularly suitable for highly dynamic scenarios such as vehicular networks; a detailed characterization of greedy routing protocols for VANETs can be found in [6]. Although very advanced protocols using, for example, traffic mobility or density information have also been proposed in the literature, this paper will consider standard geographic greedy routing protocols to demonstrate the benefits that the proposed infrastructure-based geo-routing approach can provide. Of course, nothing prevents from considering the extension of this approach to more advanced vehicular routing protocols.

Existing greedy routing protocols can be mainly classified into two main categories: position-based and topology-aware. GPSR (Greedy Perimeter Stateless Routing) [13] is one of the most commonly cited, and more simple, position-based routing protocols. In GPSR, packets generated at the source node are routed to the final destination node using positioning information. At each intermediate node, the forwarding selection process is simply based on the position of the destination node and the position of the candidate relaying neighbors (a periodic one-hop beaconing algorithm is usually used to disseminate up-to-date positioning information). By default, all nodes employ the greedy forwarding strategy and forward the data packet to the neighbour geographically closest to the destination. If a node cannot find any other neighbour closer to the destination than itself, it follows the perimeter forwarding strategy. In the Contention-Based Forwarding (CBF) protocol, intermediate nodes transmit the data packet as a single-hop broadcast message [9]. All vehicles that correctly receive the broadcast packet set a timer with its duration being proportional to their distance to the destination. As a result, the timer of the closest neighbour to the destination will expire in first place, and this node will broadcast/forward the message to be transmitted. When other nodes overhear the broadcasted message, they will reset their timers and cancel their pending forwarding transmission. In topology-aware routing protocols such as Geographic Source Routing (GSR) [15], the source node forces data packets to be routed through specific anchor 


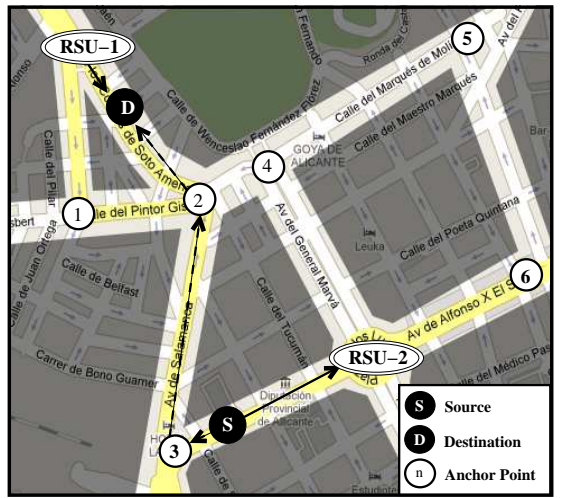

(a) City section scenario

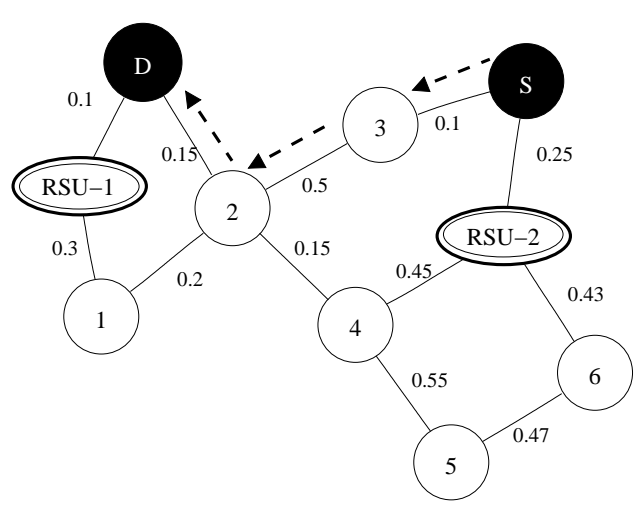

(b) Road map graph

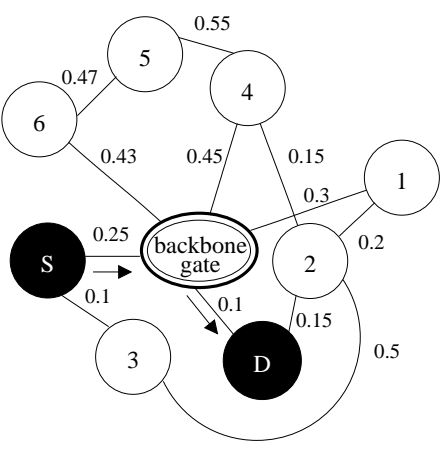

(c) Network graph

Fig. 1: City section scenario: traditional road map graph and proposed network graph. The values reported on graph edges are a qualitative example of the distance metric used in GSR

points in the path towards the destination. The forwarding process between two successive anchor points can be performed on the basis of a simple greedy position-based approach. The selection of anchor points is computed by using a planar graph representation of the street map where nodes typically represent intersections and edges represent the streets. The route of a packet is usually computed by calculating the Dijkstra shortest path between the source and the destination. It is worth noticing that the result of this computation can be embedded in the packet header at the source node or computed by each forwarding node. The GSR protocol simply weights the road map graph with the distance between two consecutive junctions. However, more sophisticated approaches have been proposed by the research community, mainly differing in the weights that are assigned to each edge or, more formally, the metric space used to calculate the shortest path between source and destination. For example, the GyTAR proposal [12] also takes into account dynamic properties, such as the vehicular traffic density, in order to select route paths that increase the probability for an end-to-end multi-hop connectivity.

\section{A. Infrastructure-Assisted Geo-Routing}

As previously explained, the use of RSUs for multi-hop communications presents two major potential advantages. In the first case, the higher antenna height increases the range and reliability of V2I communications in comparison to V2V communications. In addition, the deployed RSUs will be connected to a higher bandwidth and more reliable backbone network to enable traffic authorities the centralised access, configuration and maintenance of these units ${ }^{1}$. As a result, two RSUs can be considered to be directly connected to each other through this backbone network independently of their geographical

\footnotetext{
${ }^{1}$ Infrastructure deployments in urban scenarios would certainly benefit from existing fixed and high bandwidth backbone networks. In the case of rura or highway environments where fixed broadband connectivity might not be present, deployed infrastructure units are generally connected to traffic control centres through cellular connections. Although such cellular links generally provide lower data rates, they can provide a high transmission reliability
}

distance, which provides a valuable opportunity to improve the reliability and range of multi-hop vehicular communications. In this context, this paper aims to improve the operation, reliability and performance of multi-hop geo-routing protocols by exploiting the reliable interconnection of RSUs. Even if some latency may be added by the infrastructure, we point out that this work is mainly focused on traffic management and infotainment applications where the delay requirements are not such severe as required in safety applications. To demonstrate the benefits of the proposed infrastructure-assisted geo-routing approach, this paper uses the topology-aware GSR routing protocol, although it could certainly be extended to more advanced protocols.

The performance of topology-aware routing protocols can be improved by tailoring the metric space used by the Dijkstra algorithm, and designing a new and more complex notion of metric. Rather than designing such new metric, this paper introduces a modified graph representation, referred to as network graph, that can be directly employed by already existing topology-aware routing protocols without any modification. In the network graph, a node can represent either a road side unit or a junction. Initially, and following a traditional topology-aware approach, nodes are first connected following the road map topology and the intersection anchor points. The weights of the graph are then initially calculated following the metric employed by the considered topologyaware routing protocol, for example distance between two consecutive nodes in the case of the GSR protocol. Since RSUs can be considered to be interconnected through a reliable and high bandwidth backbone network, the geographical distance among infrastructure units should be neglected from the point of view of the geo-routing algorithm. In order to enclose this property into the network graph, all nodes that represent a RSU can be merged into a unique graph node that is referred to as backbone gate. As a consequence, vehicles will perceive all the RSUs as a unique graph node, and shortest routes can be computed using this unique property that characterises 
infrastructure nodes. To clarify the proposed infrastructureassisted geo-routing approach, Fig. 1(a) shows a city section scenario where two road side units are deployed. Traditional topology-aware routing protocols such as GSR make use of the road map graph, shown in Fig. 1(b) and with the anchor points placed at the intersections, to compute the shortest path between the source and destination nodes. In this scenario, a traditional GSR protocol would choose to forward the data packets from source to destination following the path across the nodes 2 and 3. However, if we consider that RSU-1 and RSU-2 are interconnected through a backbone network, the selected GSR path is non-optimal since the shortest path would then be through the two RSUs. The scenario and graph depicted in Fig. 1(b) represent then a clear example under which the potential benefits of the infrastructure would not be efficiently used. To exploit the use of the infrastructure units, Fig. 1(c) shows our proposed network graph where the two road side units are merged into a unique node due to their interconnection through a backbone network. Using this new graph representation, topology-aware routing protocols would be able to compute more optimal ${ }^{2}$ routes and, when it is the case, efficiently route packets through the infrastructure. It has to be noted that the concepts of backbone gate and virtual equivalent node, introduced in [11], can appear really similar at first glance; nevertheless the two concepts substantially differ from each other because the virtual equivalent node doesn't take part as a decision factor in the route discovery process.

\section{Simulation ENVIRONMENT}

The performance of the proposed infrastructure-assisted routing approach has been evaluated using the ns-2 simulator, and SUMO (Simulation of Urban Mobility) to generate vehicular mobility traces. This work considers a 1000x1000 $m^{2}$ Manhattan-like urban scenario as depicted in Fig. 2 under three different traffic conditions, hereinafter referred to as Manhattan-A, Manhattan-B, and Manhattan- $C$. Fig. 2 shows a qualitative representation of the normalized average vehicular density for the three considered urban Manhattan-type scenar$\operatorname{ios}^{3}$. The scenarios consider low capacity (with only one lane for each direction) and high capacity roads (with three lanes for each direction). While the Manhattan-A scenario is only based on low capacity roads, the Manhattan-B and Manhattan$C$ scenarios consider both low and high capacity roads ${ }^{4}$. This allows analysing the impact of the proposed infrastructureassisted routing approach under uniform and non-uniform vehicular conditions. Vehicular traffic is generated using SUMO with vehicles entering the simulated scenario uniformly in time during the whole simulation period. The Manhattantype scenarios have been simulated considering four different vehicular densities representing low, medium, high, and very high density conditions (see Table I). Although the same number of vehicles are emulated for each traffic density, the

\footnotetext{
${ }^{2}$ In the example shown in Figure 1, optimal refers to shortest routes.

${ }^{3}$ The normalization is performed over the maximum measured vehicular density for each scenario.

${ }^{4}$ The rationale under the election of the high capacity roads will be clarified in Section IV.
}

TABLE I: Average vehicular density [vehicles/Km/lane]

\begin{tabular}{|c||c|c|c|c|}
\hline & low & medium & high & very high \\
\hline \hline Manhattan- $A$ & 2.22 & 4.35 & 7.01 & 12.52 \\
\hline Manhattan- $B$ & 2.26 & 3.62 & 4.97 & 7.27 \\
\hline Manhattan- $C$ & 2.25 & 3.57 & 4.84 & 6.57 \\
\hline
\end{tabular}

specific average vehicular density varies per scenario due to the different selection/deployment of low and high capacity roads depicted in Fig. 2 that influences the vehicular mobility patterns. In the simulated scenarios, all junctions are regulated by the 'right-before-left' traffic priority rule where vehicles coming from the right side have the right to go first. Vehicles can reach a maximum speed of $50 \mathrm{~km} / \mathrm{h}$, and vehicular micromobility is regulated by the Krauß car-following model [14].

Vehicles communicate using the IEEE 802.11 p or ITSG5A standard. In particular, vehicles periodically broadcast Cooperative Awareness Messages (CAMs), also known as beacons, with a $2 \mathrm{~Hz}$ frequency and a data rate equal to $6 \mathrm{Mb} / \mathrm{s}$. Such CAM messages include positioning data that allows each vehicle to be aware of the position of its 1-hop neighbours. To analyse the performance of geo-routing protocols, data packets are transmitted between source and destination vehicular nodes that are randomly chosen for each data packet. Data packets are generated with $10 \mathrm{~Hz}$ frequency, and the size of each packet has been set to 512 bytes (including a header of 84 bytes and a 428 bytes payload); the payload size has been derived from [8] where packets are composed by a security/authentication header of 200 bytes and by many optional fields depending on the different applications running on each vehicle. Each relay node in the transmission of the data packet from source to destination introduces a processing delay of $50 \mathrm{~ms}$. The maximum delay $\delta_{C B F}$ used to schedule the re-broadcast of a packet in $\mathrm{CBF}$ has been set equal to $0.8 \mathrm{~s}$. As we will discuss later, the fine tuning of this parameter depends on the average vehicular density, and can have an impact on the CBF performance.

Recent studies have demonstrated the need to use accurate radio propagation models to extract valid conclusions regarding the performance and operation of routing protocols for vehicular ad-hoc networks [4]. In this context, this work is based on the urban micro-cell radio propagation model derived during the European WINNER project for the $5 \mathrm{GHz}$ band [21]. Although not specifically developed for $\mathrm{V} 2 \mathrm{~V}$ communications, to the authors knowledge, the operating conditions of the WINNER urban micro-cell model are those that currently best fit the V2V and V2I communications scenario. The model tries to account for the effects of pathloss, shadowing and multipath fading. While pathloss represents the local average received signal power relative to the transmit power as a function of the distance between transmitter and receiver, the shadowing models the effect of surrounding obstacles on the mean signal attenuation. The multipath fading effect results from the reception of multiple replicas of the transmitted signal at the receiver. Another key aspect of this propagation model that is of considerable interest for urban scenarios is that it differentiates between Line of Sight (LOS) and Non Line 


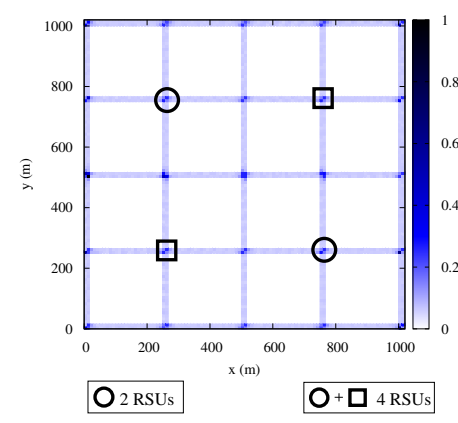

(a) Manhattan-A

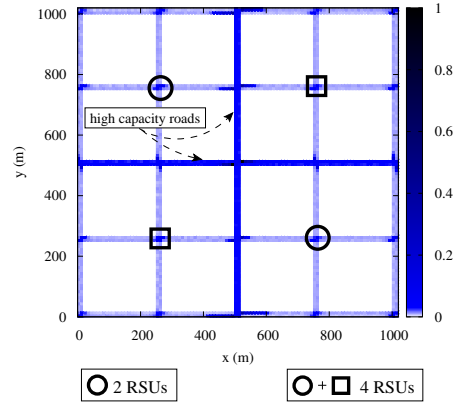

(b) Manhattan-B

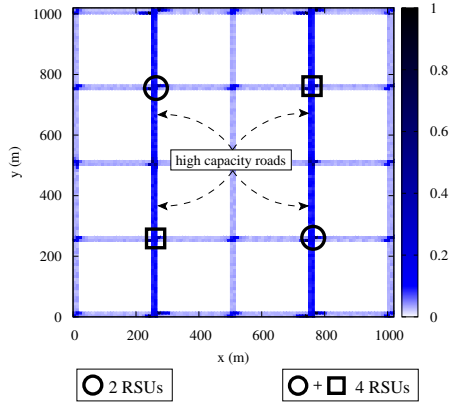

(c) Manhattan-C

Fig. 2: Manhattan-like scenarios with different topology and vehicular mobility (squares and circles indicate the position of road side units)

of Sight (NLOS) conditions. The pathloss model for LOS conditions can be expressed as follows:

$P L_{L O S}(d[m])=\left\{\begin{array}{l}22.7 \cdot \log _{10}(d)+41+ \\ +20 \cdot \log _{10}(f[G H z] / 5) \text { if } d<R_{b p} \\ 40 \cdot \log _{10}(d)+41-17.3 \log _{10}\left(R_{b p}\right)+ \\ +20 \cdot \log _{10}(f[G H z] / 5) \text { if } d \geq R_{b p}\end{array}\right.$

where,

$$
R_{b p}=4 \cdot \frac{\left(h_{A}-1\right) \cdot\left(h_{B}-1\right)}{\lambda}
$$

and $h_{A}$ and $h_{B}$ are the heights of receiver and transmitter antennas respectively (in meters).

For NLOS conditions, the path-loss can be expressed as:

$$
\begin{aligned}
P L_{N L O S}\left(d_{A}[m], d_{B}[m]\right)= & P L_{N L O S}\left(d_{A}\right)+ \\
& +20-12.5 \cdot \nu_{j}+ \\
& +10 \cdot \nu_{j} \cdot \log _{10}\left(d_{B}\right)
\end{aligned}
$$

where,

$$
\nu_{j}=\max \left(2.8-0.0024 d_{A}, 1.84\right)
$$

and $d_{A}$ and $d_{B}$ are the transmitter and receiver distances to the closest intersection (in meters).

The shadowing effect is modelled with a log-normal random distribution with standard deviation equal to $3 \mathrm{~dB}$ and $4 \mathrm{~dB}$ for LOS and NLOS conditions respectively in urban microcell scenarios [21]. To account for the shadowing spatial correlation, the Gudmundson model considering an exponential autocorrelation function [10] is employed in this work. Finally, the multipath fading effect has been modelled as a Ricean distribution for LOS and as a Rayleigh one for NLOS conditions [21]. In addition to propagation loses, this work models the probabilistic nature resulting from radio transmission effects through the inclusion of the PER (Packet Error Rate) performance as a function of the Signal to Interference and Noise Ratio (SINR) [22].

\section{Performance Evaluation}

As explained in Section II, the proposed network graph representation could be smoothly employed by any of the already existing topology aware routing protocols. As a first evaluation step, GSR has been elected as the reference topology aware routing protocol over which to test the infrastructure-assisted approach proposed in this paper. The GSR protocol has been selected due to its low complexity and flexibility. In this context, it is then interesting to analyse whether a simple geo-routing protocol using the proposed infrastructure-assisted routing approach can achieve the same performance as more dynamic protocols. To this aim, the performance of GSR and the proposed infrastructure-assisted routing approach are compared against that achieved with two commonly accepted position-based routing protocols, GPSR and CBF. Following the evaluation scenarios depicted in Fig. 2, the infrastructureassisted GSR routing protocol is referred as GSR-2 and GSR4 , depending on whether 2 or 4 RSUs are deployed in the Manhattan-type scenario. To evaluate the performance of the different geo-routing protocols, the following performance metrics are used:

- Packet Delivery Ratio: defined as the ratio between packets successfully delivered to their destination and all the packets generated at the source nodes;

- Overhead: defined as the number of bytes generated by the routing protocols for each packet generated at a source node;

- Wireless Hops: defined as the average number of wireless hops needed to successfully deliver a packet from source to destination;

- Geographic distance: defined as the average geographic distance between source and destination nodes when packets successfully reach the destination node.

\section{A. Uniform Traffic Density}

The performance of the different routing protocols has been evaluated under uniform and non-uniform traffic densities as reported in Section III. This section analyses first their performance under uniform traffic conditions, which corresponds to the Manhattan- $A$ scenario depicted in Figure 2(a). Fig. 3 shows the packet delivery ratio for this uniform traffic 


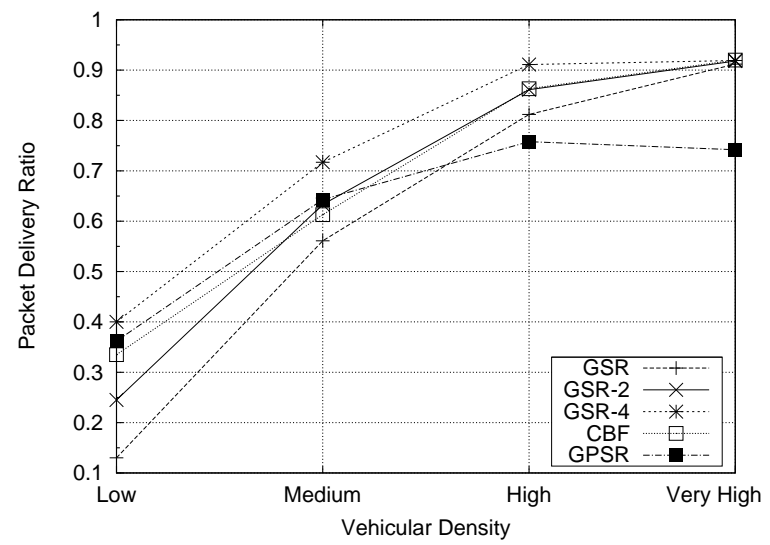

Fig. 3: Packet delivery ratio under uniform traffic conditions (Manhattan-A)

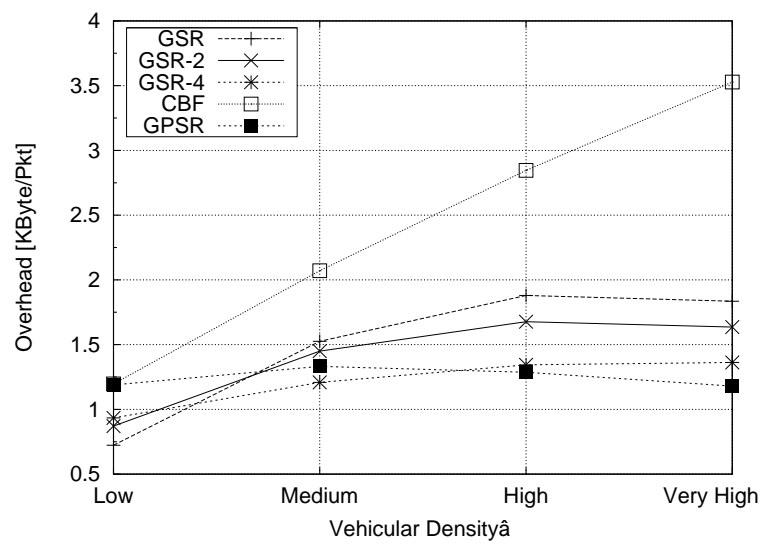

Fig. 4: Protocol overhead under uniform traffic conditions (Manhattan-A)

scenario for different vehicle densities. As expected, CBF outperforms GPSR and GSR for high and very high densities. It is important to note that the degradation of CBF for low and medium densities is mainly caused by the well-known problems of local minimums and packet deletion effects [9]. Even though a comprehensive performance evaluation of CBF is out of the scope of this paper, it should be noted that these undesirable effects could be partially mitigated by a fine tuning of the maximum scheduling delay $\delta_{C B F}$; the value of $\delta_{C B F}$ used in our simulations has been empirically optimized for high densities. The results depicted in Fig. 3 show that using the proposed infrastructure-assisted routing approach with just 2 RSUs is sufficient for the GSR protocol to achieve the same performance as the CBF protocol for medium and high densities. In addition, the proposed infrastructure-assisted routing approach outperforms the other simulated protocols independently of the vehicular densities when deploying 4 RSUs in the simulated scenario. To better understand the functionality and potential benefits of an infrastructure-assisted routing approach, it is interesting to analyse some operational parameters. Fig. 4 depicts the overhead generated by each protocol as a function of the vehicular density. The overhead is computed as the number of routing packets generated between a successful source-destination data transmission, multiplied

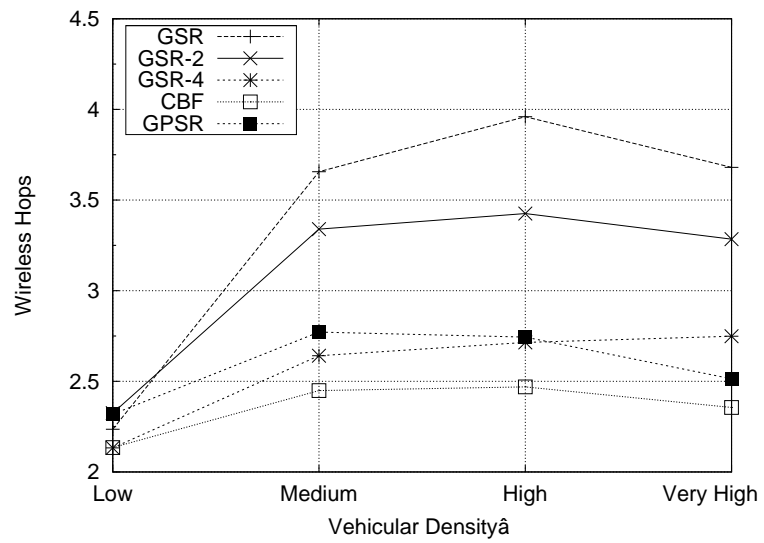

Fig. 5: Wireless hops under uniform traffic conditions (Manhattan-A)

by the size of such packets which has been assumed to be equal to 512bytes. As it can be observed, the introduction of the infrastructure-assisted routing approach enables reducing the routing overhead, with the reduction increasing as the number of deployed road side infrastructure units increases. This routing overhead reduction is due to a lower number of average hops (see Fig. 5) needed to successfully deliver a packet to the destination node. The results depicted in Fig. 5 also show that $\mathrm{CBF}$ is the protocol requiring a lower number of average hops to reach the destination node. However, this protocol is characterised by a larger routing overhead (Fig. 4). As it has been reported in [4], the broadcast nature of the CBF protocol can frequently induce packet duplications in urban environments due to the presence of obstacles and Non Line of Sight (NLOS) propagation conditions that prevent vehicles to overhear the forwarding of a data packet by another nearby vehicle. Such duplication is at the origin of the larger routing overhead of the CBF protocol depicted in Fig. 4. Another interesting property of the infrastructure-assisted routing approach is that it enables the establishment of larger distance multi-hop vehicular communications (see Fig. 6) compared to the other simulated protocols. This trend is maintained independently of the vehicular density, although the geographical scalability of the infrastructure-assisted approach depends on the number of deployed RSUs.

\section{B. Non-Uniform Traffic Density}

The previous results have highlighted the potential of the proposed infrastructure-assisted routing approach to increase the end-to-end performance of multi-hop vehicular communications, while also reducing the communications overhead. However, the previous results considered a uniform traffic density scenario, and it is therefore necessary to analyse whether such performance benefits can still be maintained under non-uniform traffic conditions. The results depicted in Fig. 7 correspond to the packet delivery ratio achieved under non-uniform traffic conditions following the Manhattan$B$ scenario illustrated in Fig. 2(b). The obtained results confirm that the infrastructure-assisted routing approach is capable to increase the performance of simple topology-aware routing 


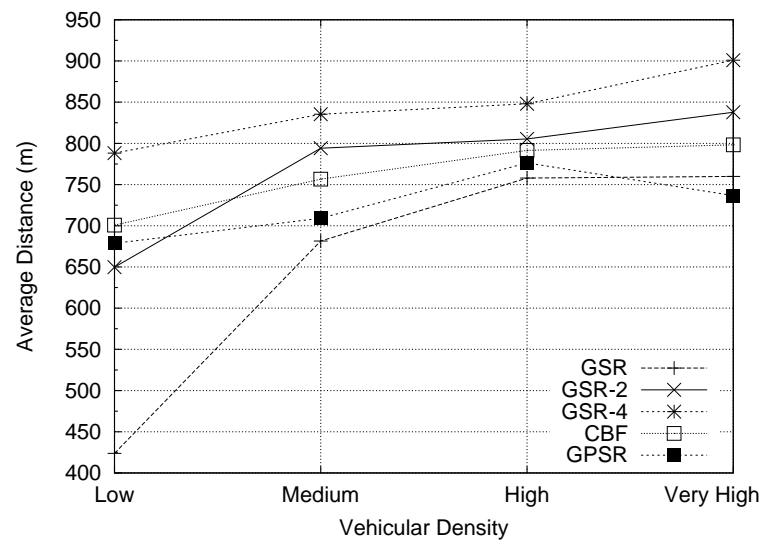

Fig. 6: Average geographic distance under uniform traffic conditions (Manhattan-A)

protocols such as GSR. Despite such improvements, CBF outperforms the proposed approach for all vehicular densities. This trend is due to a non-optimal deployment of the RSUs with respect to the vehicular density that prevents from fully exploiting their routing benefits as observed in Fig. 8. This figure represents the usage probability of the deployed RSUs in the routing process from source to destination. The depicted results clearly show that the lower performance of the proposed infrastructure-assisted routing approach is due to a lower routing usage of the infrastructure units in the Manhattan- $B$ scenario with respect to the Manhattan- $A$ one. These results clearly show that to obtain the maximum benefits from the proposed infrastructure-assisted routing approach, an optimal deployment of RSUs must be considered. To emphasize such dependence, the infrastructure units are deployed in the higher density road segments in the Manhattan- $C$ scenario illustrated in Fig. 2(c). In this new scenario, the packet delivery ratio of the proposed infrastructure-assisted routing approach outperforms again the other simulated routing protocols due to a higher usage of the RSUs in the routing process from source to destination ${ }^{5}$. Although the design of an optimal deployment procedure is out of the scope of this paper, and is left for future work, it is important to note that such deployment strategy should not only consider the vehicular density, but also the routing diversity that can be obtained from the deployment and usage of infrastructure units.

\section{CONCLUSIONS}

This paper has presented and evaluated a novel infrastructure-assisted routing approach for cooperative vehicular networks. The proposed approach introduces a simple and new graph representation of the road-topology map that takes into account the relaying capabilities of road side infrastructure units for multi-hop vehicular communications, and that can be applied to existing topology-aware routing protocols. The conducted study has shown that the proposed

\footnotetext{
${ }^{5}$ As observed for uniform traffic scenarios, the proposed infrastructureassisted routing approach also reduces the communications overhead, and is capable to increase the geographical distance between source and destination nodes under non-uniform traffic conditions
}

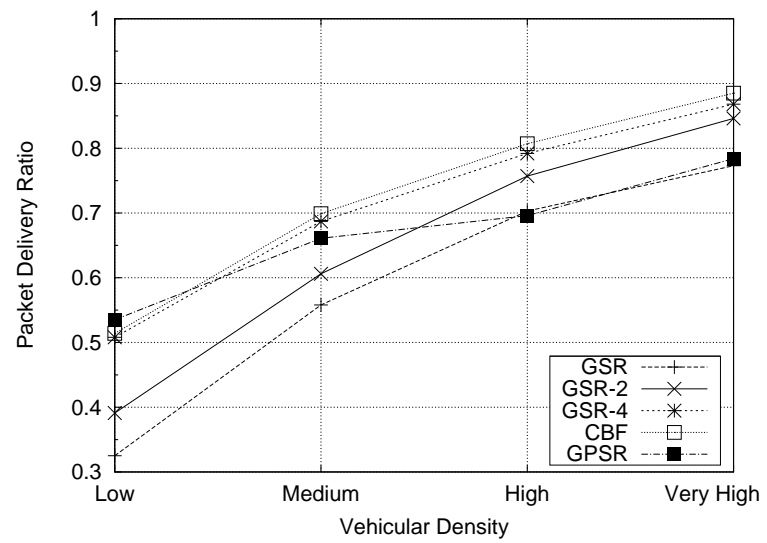

Fig. 7: Packet delivery ratio under non-uniform traffic conditions (Manhattan-B)

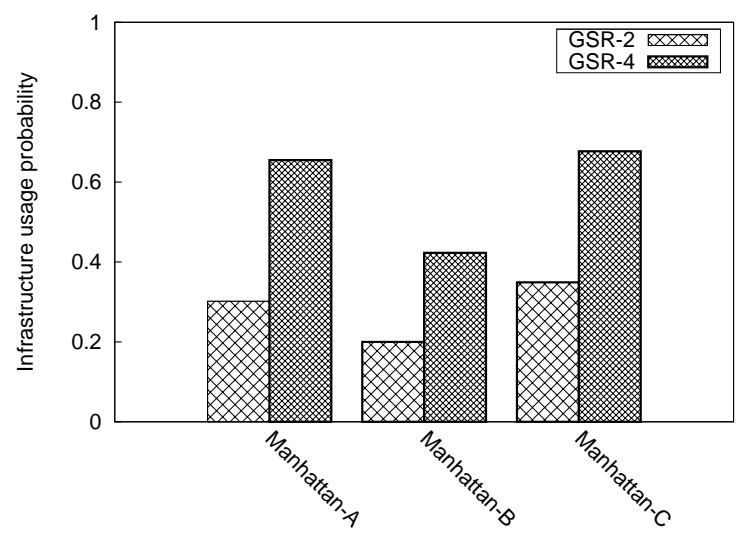

Fig. 8: Infrastructure usage probability

approach can improve the packet delivery ratio and increase the communications distance between source and destination nodes, while reducing the communications overhead. This study has also shown that the benefits of the proposed infrastructure-assisted routing approach partially depend on the vehicular distribution and the infrastructure deployment strategy. As a result, the authors are currently investigating optimal infrastructure deployment methods that will allow

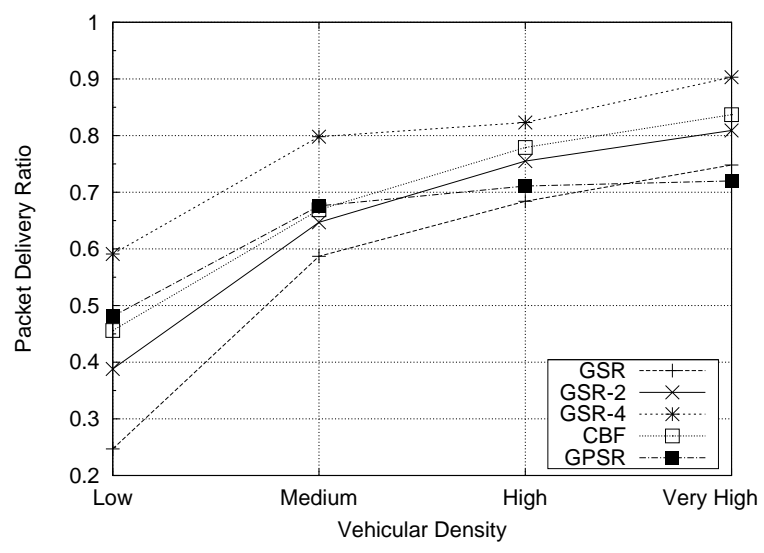

Fig. 9: Packet delivery ratio under non-uniform traffic conditions (Manhattan-C) 
fully exploiting the characteristics and benefits of road side infrastructure units in cooperative vehicular communications.

\section{ACKNOWLEDGMENTS}

This work has been partly funded by the European Commission through FP7 ICT Project iTETRIS: An Integrated Wireless and Traffic Platform for Real-Time Road Traffic Management Solutions (No. FP7 224644). The authors wish to acknowledge the Commission for their support.

\section{REFERENCES}

[1] B. Aslam, Ping Wang, and C. Zou. An economical, deployable and secure vehicular ad hoc network. In Proc. IEEE Military Communications Conference, pages 1-7, November 2008.

[2] B. Baldessari, A. Festag, A. Matos, J. Santos, and R. Aguiar. Flexible connectivity management in vehicular communication networks. In Proc. of International Workshop on Intelligent Transportation, March 2006.

[3] S. Barghi, A. Benslimane, and C. Assi. A lifetime-based routing protocol for connecting vanets to the internet. In Proc. of IEEE International Symposium on a World of Wireless, pages 1-9, June 2009.

[4] R. Bauza, J. Gozalvez, and M. Sepulcre. Operation and performance of vehicular ad-hoc routing protocols in realistic environments. In Proc. of IEEE International Symposium on Wireless Vehicular Communications, pages 1-5, September 2008.

[5] M. Bechler and L. Wolf. Mobility management for vehicular ad hoc networks. In Proc. of IEEE Vehicular Technology Conference, pages 2294-2298, May 2005.

[6] J. Bernsen and D. Manivannan. Greedy routing protocols for vehicular ad hoc networks. In Proc. of Wireless Communications and Mobile Computing Conference, pages 632-637, August 2008.

[7] S.S Chawathe. Inter-vehicle data dissemination in sparse equipped traffic. In Proc. of Intelligent Transportation Systems Conference, pages 273 - 280, September 2006.

[8] COMeSafety. D31: European its communication architecture: Overall framework proof of concept implementation. In COMeSafety Public Deliverable, March 2009.

[9] H. Füßler, J. Widmer, M. Ksemann, M. Mauve, and H. Hartenstein. Contention-based forwarding for mobile ad hoc networks. In Elsevier Ad Hoc Networks Journal, pages 351-369, November 2003.

[10] M. Gudmundson. Correlation model for shadow fading in mobile radio systems. In Electronic Letters, pages 2145-2146, November 1991, 27 (23).

[11] Rongxi He, H. Rutagemwa, and Xuemin Shen. Differentiated reliable routing in hybrid vehicular ad-hoc networks. In Proc. of IEEE International Conference on Communications, pages 2353-2358, May 2008.

[12] M. Jerbi, S.-M. Senouci, R. Meraihi, and Y. Ghamri-Doudane. An improved vehicular ad hoc routing protocol for city environments. In Proc. of IEEE International Conference on Communications, pages 3972-3979, June 2007.

[13] B. Karp and H. T. Kung. Gpsr: greedy perimeter stateless routing for wireless networks. In Proc. of the 6th annual international conference on Mobile computing and networking, ACM,, pages 243-254, August 2000.

[14] S. Krauss, P. Wagner, and C. Gawron. Metastable states in a microscopic model of traffic flow. In Physical Review, Vol. 55, pages 5597-5602, May 1997.

[15] C. Lochert, H. Hartenstein, J. Tian, H. Fussler, D. Hermann, and M. Mauve. A routing strategy for vehicular ad hoc networks in city environments. In Proc. of IEEE Intelligent Vehicles Symposium, pages 156-161, June 2003.

[16] C. Lochert, B. Scheuermann, M. Caliskan, and M. Mauve. The feasibility of information dissemination in vehicular ad-hoc networks. In Proc. of the 4th Fourth Annual Conference on Wireless on Demand Network Systems and Services, pages 92-99, January 2007.

[17] C. Lochert, B. Scheuermann, C. Wewetzer, A. Luebke, and M. Mauve. Data aggregation and roadside unit placement for a vanet traffic information system. In VANET 2008: Proceedings of the Fifth ACM International Workshop on VehiculAr Inter-NETworking, pages 58-65, September 2008.

[18] Yanlin Peng, Z. Abichar, and J.M. Chang. Roadside-aided routing (rar) in vehicular networks. In Proc. of IEEE International Conference on Communications, pages 3602-3607, June 2006.
[19] R.K. Shrestha, Sangman Moh, Ilyong Chung, and Dongmin Choi Vertex-based multihop vehicle-to-infrastructure routing for vehicular ad hoc networks. In Proc. of 43rd Hawaii International Conference on System Sciences, pages 1-7, January 2010.

[20] O. Trullols, M. Fiore, C. Casetti, C. F. Chiasserini, and J. M. Barcelo Ordinas. Planning roadside infrastructure for information dissemination in intelligent transportation systems. In Computer Communications, pages 432-442, 2010, 33 (4).

[21] WINNER. D1.1.1. winner ii interim channel models. In Public deliverable: http://www.ist-winner.org/.

[22] A. Zang. An error model for inter-vehicle communications in high-way scenarios at 5.9ghz. In Proc. of Workshop on Performance evaluation of wireless ad hoc, sensor, and ubiquitous networks, pages 49-56, October 2005.

[23] J. Zhao, Y. Zhang, and G. Cao. Data pouring and buffering on the road: A new data dissemination paradigm for vehicular ad hoc networks. In IEEE Transactions on Vehicular Technology, pages 32663277, November 2007, 56 (6). 